\title{
Afibrinogenemia en el embarazo
}

\author{
Dr. Rafael Quiñones Daza
}

La literatura médica reciente nos trae un sinnúmero de estudios sobre la diátesis hemorrágica que en algunos casos de embarazo se presenta, producida por el síndrome de "Afibrinogenemia adquirida" y que se asocia principalmente a tres entidades obstétricas: 1) Casos severos de desprendimiento prematuro de la placenta; 2) Embolía o "infusión" del líquido amniótico y 3) Retención prolongada del feto muerto, especialmente en pacientes $\mathrm{Rh}$ negativas que poseen aglutininas anti-Rh.

Como en los Estados Unidos tuve oportunidad de seguir de cerca algunos de estos casos y puede apreciar la forma en que los hospitales se están preparando para garantizar un diagnóstico precoz y un tratamiento inmediato de esta entidad, quiero en este trabajo reúnir lo más importante de la información dispersa de muchas revistas, presentar dos casos clínicos y hacer algunas anotaciones sobre la necesidad de garantizar en Bogotá el tratamiento adecuado y rápido que este síndrome exige.

\section{HISTORIA}

En 1901, De Lee al reportar un caso de desprendimiento prematuro de la placenta con hemorragia incontrolable, se refiere por primera vez a un trastorno en la coagulación de la sangre asociado con esta entidad obstétrica y lo describe como una "hemofilia pasajera". Anota este autor que la sangre de la enferma permanecería incoagulable aún después de varios días. Williams en 1915, se refiere a un caso similar acompañado de hemorragia fatal y cree en la existencia de un principio tóxico parecido al trasmitido por la mordedura de serpientes. Siete años después Wilson propone la teoría de que cierto material tóxico o hemorragina pasaría de la placenta a la circulación materna para producir esta falla en la coagulación sanguínea y presenta 14 casos de 
muerte por hemorragia continua después de desprendimiento prematuro de la placenta.

Simultáneamente se desarrollan investigaciones orientadas hacia la toxicidad de extractos placentarios y en 1919 Obata, al inyectar extractos acuosos de placenta a conejos por vía endovenosa, encuentra primero un aceleramiento y luego una demora en la coagulación sanguínea. Hori, Sakurai y Schneider confirman lo anterior y este último encuentra que la tromboplastina es el principio tóxico que actúa sobre la sangre de los conejos y anota que los animales que sobreviven a las dosis no letales de extractos placentarios, se hacen refractarios a las dosis letales.

Dieckmann en 1936 nota marcada reducción en el nivel de fibrinógeno en pacientes que sangraban después de desprendimiento prematuro de la placenta y en algunos casos fatales de eclampsia. Fulton, Page y Kellogg en 1948, presentan más casos de hemorragia producida por la placenta "abruptio" y encuentran que la falla en la coagulación era causada por defibrinación debida al paso de tromboplastina a la circulación materna. Chargaff identifica también a la trombosis como el factor coagulante contenido en extractos salinos de placenta. En 1945, Smith y Smith ya habían descubierto actividad fibrinolítica en el suero sanguíneo de algunas enfermas con toxemia del embarazo. En 1950. Moloney reporta el primer caso de tratamiento efectivo de afibrinogenemia consecutiva a placenta "abruptio" con fibrinógeno humano y él lo mismo que Weiner y Reid atribuyen la causa de este trastorno a una enzima fibrinolitica. En el mismo año, Schneider produce experimentalmente desprendimientos de la placenta en conejos traumatizando el sitio de inserción de ésta y atribuye las alteraciones viscerales que se producen, a la tromboplastina que se origina en el lugar del traumatismo y que sería recogida por la sangre materna.

Otra fase de la historia de este sindrome la podemos observar desde 1941, cuando stewer y Lushbaugh describieron como la mayor causa de mortalidad materna durante el trabajo la embolía por líquido amniótico. Originalmente atribuyeron la causa de la muerte en estos casos a los efectos embólicos del líquido amniótico asociados con una reacción de tipo anafiláctico. En posteriores comunicaciones se observó que las enfermas que sobrevivían al Shock inicial por la embolía, presentaban hemorragias en las membranas mucosas y en la superficie de los órganos y otras desarrollaban hemorragias uterinas, Reid, Weiner y Raly en 
comunicación de septiembre de 1953 y después de estudiar los mecanismos de lo coagulación en el embarazo y de revisar las historias de muchas pacientes muertas debido a hemorragias incontrolables, encuentran que el factor decisivo es el carácter de esa sangre de ser absolutamente incoagulable y además advierten que la presencia de líquido amniótico en la circulación materna puede estar asociada a ese trastorno en la coagulación sanguínea. Continuando los estudios al respecto, encuentran en el líquido amniótico una sustancia similar a la tromboplastina y logran reproducir el síndrome inyectando este líquido a conejos. Proponen estos autores la tesis de que el curso clínico y la muerte en los casos de embolía por líquido amniótico son debidos a coagulación intravascular y defibrinación y afirman que este síndrome puede ser corregido con la administración de fibrinógeno y sangre total fresca.

Los mismos autores ya nombrados, Weiner, Reid y Raly, han investigado desde 1946 en el Boston Lying-in Hospital el síndrome hemorrágico presentado por algunas pacientes $\mathrm{Rh}$ negativas con aglutininas anti-Rh y quienes habian hecho una prolongada retención del feto muerto, y han llegado a la conclusión de que el defecto en la coagulación de la sangre de estas enfermas es similar al que se asocia con desprendimiento prematuro de la placnta y embolía por líquido amniótico; afirman ellos que es debido a una afibrinogenemia adquirida, posiblemente producida por coagulación intravascular provocada por una sustancia coagulante que derivaría de la autolisis de la decidua y el tejido placentario.

\section{CARACTERISTICAS CLINICAS}

Varios nombres se han sugerido para calificar el sindrome que vamos a estudiar, entre ellos los de Hipofibrinogenia, fibrinogenopenia y anemia afibrinogenética, pero nosotros usaremos el más aceptado por los autores americanos, afibrinogenemia y que es talvez el más adecuado dada la desaparición casi total del fibrinogeno circulante que se observa en la mayoría de los casos.

Este síndrome se presenta en algunos casos de embarazo, asociado principalmente a 3 entidades: 1) Desprendimiento prematuro de la placenta; 2) Embolía o "infusión" de líquido amniótico, y 3) Retención prolongada del feto muerto, especialmente en pacientes $R h$ negativas que poseen aglutininas antiRh. Se han descrito algunos pocos casos aislados en Toxemias 
muy severas del embarazo y en casos de Shock obstétrico no relacionado con ninguna de las entidades anteriores, pero ellos no se han aceptado como manifestaciones clásicas de este síndrome.

Hematológicamente se manifiesta por una marcada deplesión del fibrinógeno circulante en el plasma, debida a coagulación intravascular diseminada, lo cual va a producir hemorragias que no se pueden controlar por los medios ordinarios (transfusiones, medicación coagulante, etc.). La característica clínica principal es la incapacidad absoluta de la sangre de estas pacientes para coagularse una vez fuera del organismo. Estas hemorragias se pueden presentar en superficies cruentas como lo son el sitio de inserción de la placenta, las incisiones de episiotomías, las incisiones uterinas y de la pared abdominal para operaciones cesáreas, pero pueden ocurrir también en las membranas mucosas intactas o ser subperitonales como en el caso de las "lesiones de Couvelaire" que algunas veces acompañan a los desprendimientos prematuros de la placenta.

Aunque la caractersítica clínica del síndrome es una sola. incapacidad de la sangre para coagularse y producción de hemorragias incontrolables, el cuadro variará ligeramente en las 3 entidades obstétricas ya nombradas, por lo cual nos referimos a cada una de ellas por separado.

1) Desprendimiento prematuro de la placenta.- El cuadro clínico presentado en estos casos será el propio de esta entidad: dolor abdominal, contracciones uterinas muy fuertes y prolongadas o útero tetánico desvanecimiento de los ruidos fetales o total desaparición de ellos y hemorragia vaginal. La enferma mostrará distintos grados de Shock de acuerdo con la cantidad de sangre que esté perdiendo, con la consiguiente caída de la tensión arterial, aceleración del pulso, palidez, etc. El sindrome de afibrinogenemia se habrá establecido cuando se observe la tendencia de la sangre a no coagularse y cuando se vea que la hemorragia cada vez más abundante no cede a ninguno de los tratamientos ordinarios. A estas enfermas podrán suministrárseles innumerables transfusiones de sangre total, se les podrá provocar el parto, ya sea vaginalmente por inducción o por medio de operación cesárea y seguirán sangrando continuamente mientras no se les administre el único tratamiento racional que es el fibrinógeno humano o "fracción 1 de Cohn". Al darles este tratamiento se notará en muy pocos minutos que la hemorragia ya ha cedido y que la sangre comienza a formar coágulos. Al leer la literatura, 
al respecto podemos ver casos en que se han trasfundido hasta 34 botellas de sangre en un periodo de ocho horas, logrando tan solo mejorar el estado general y el grado de Shock, pero sin lograr contener la diátesis hemorrágica.

El sindrome puede presentarse o bien antes del parto cuando aparezcan los primeros signos de desprendimiento de la placenta o en cualquier momento del puerperio, poco después del alumbramiento o de la operación cesárea, o varias horas o días después. Puede caracterizarse únicamente por hemorragia uterina 0 puede presentarse en forma de hemorragia proveniente de la herida de episiotomía o de la insición hecha para operación cesárea y en algunas ocasiones por hemorragias gástricas, epistaxis incontrolables, etc. Estas enfermas sangran fácilmente por el sitio en donde se han aplicado inyecciones intravenosas y hacen hematomas subcutáneos y manchas equimóticas diseminadas, en fin, todas las manifestaciones de una diátesis hemorrágica muy severa. Cuando la hemorragia post-partum es uterina llamará la atención el buen tono y contractilidad de la matriz.

Se ha observado que el síndrome de afibrinogenemia se presenta únicamente en algunos casos muy severos de desprendimiento prematuro de la placenta y que la alteración en la coagulación de la sangre no precede sino que sigue al desprendimiento.

2) Embolia o infusión de liquido amníótico.- Este síndrome descrito por primera vez por Steiner y Lushbaugh en 1941, presenta en la mayoría de las veces el siguiente cuadro clínico: durante el trabajo o en el momento del parto la paciente presenta súbitamente una crisis de cianosis, en ocasiones presenta vómito o convulsiones de tipo anóxico e inmediatamente después entra en estado de profundo Shock con descenso marcado de la tensión arterial, aceleración y debilitamiento del pulso, etc. Algunas de estas pacientes mueren antes de nacer el niño, mientras que las que sobreviven al Shock inicial por lo general sucumben algunas horas después. En este último grupo se aprecian equímosis diseminadas y hemorragia uterina o proveniente de las mucosas intactas; a la autopsia de estas pacientes además de encontrar en los vasos pulmonares las lesiones embólicas de contenido amniótico, se encuentran múltiples hemorragias internas, especialmente en la superficie de los órganos. Desde los estudios de Reid, Weiner y Roby, quienes fueron los primeros en encontrar una relación entre la embolía del líquido amniótico y el síndrome de afibrinogenemia, se han reportado muchos más casos y en todos ellos 
llama la atención el carácter de incoagulabilidad de la sangre de esas pacientes. Como en los casos de placenta "abruptio" la administración de transfusiones mejorará en algo el estado general, pero la restauración del fibrógeno circulante y de los mecanismos de coagulación de la sangre no se logrará sino con la administración de fibrinógeno.

Al revisar todos estos casos los autores, anotan que el grado de bloqueo mecánico de los vasos pulmonares por émbolos de líquido amniótico no alcanza a explicar la muerte de estas pacientes y sugieren que en muchas de estas muertes la causa principal sería el trastorno en la coagulación de la sangre con sus consiguientes hemorragias internas y externas y la formación de depósitos de fibrina por coagulación intravascular de la sangre. Numerosos reportes se conocen ya de tratamiento eficaz con fibrinógeno cuando se ha hecho un diagnóstico precoz, por lo demás muy simple.

Se ha notado que estas embolías por líquido amniótico se presentan con mayor frecuencia en casos de ruptura prematura de las membranas seguida de un trabajo muy fuerte y rápido. Parece que únicamente se presentan en casos en que las contracciones uterinas son de una intensidad mayor que la ordinaria y se han descrito varios casos en trabajos inducidos por pitocín, en los cuales esta droga ha provocado contracciones particularmente vigorosas.

3) Retención prolongada de feto muerto.- Weiner observó por primera vez en 1946 en el Boston Lying-in Hospital un caso de retención prolongada de feto muerto asociado con cierta diátesis hemorrágica. Este mismo autor, Ratnoff y Peckhan han seguido investigando esta rara asociación y han deportado numerosos casos. Se ha llegado a la conclusión de que en muchas pacientes $\mathrm{Rh}$ negativas que posean un buen título de aglutininas anti$\mathrm{Rh}$ y que llegan a retener el feto muerto por algún tiempo se presenta el síndrome de afibrinogenemia con la consiguiente diátesis hemorrágica. En estas pacientes se manifiesta en muchos casos por hemorragias gingivales o nasales y equímosis cutáneas y en otras por hemorragias vaginales antes o después del parto. La sangre perdida por estas pacientes tendrá la misma característica de ser incoagulable y este defecto no se corregirá totalmente sino por la pronta administración de fibrinógeno.

Aunque la mayoría de los casos reportados se presentan en enfermas con incompatibilidad $R h$, ya se han visto varios en los cuales no existe ninguna incompatibilidad de Tipo o de Grupo. 
En el mismo hospital americano ya nombrado se han llevado a cabo los estudios más serios al respecto y se ha llegado a hacer el diagnóstico antes de la aparición clínica del síndrome, midiendo los niveles de fibrinógeno en la sangre y se han reportado muchos casos de prevención y de curación del síndrome con la administración de fibrinógeno.

En la mayoría de estos casos, el feto muerto se ha retenido por más de un mes y en ellos no se ha notado ninguna asociación de estas hemorragias con embolias de líquido amniótico como desprendimiento prematuro de lá placenta. En los casos en que no se ha obtenido el tratamiento adecuado, las enfermas entran en Shock por la enorme pérdida de sangre y a pesar de innumerables transfusiones histerectomía, etc., la mayoría de ellas mueren. En estos, como en los casos anteriormente descritos se notará la coexistencia de hemorragias post-partum incontrolables provenientes de un útero bien contraido y firme, y la ausencia de desgarros o lesiones que justifiquen la hemorragia.

\section{PATOGENIA}

Aunque son muchas las teorías que se han dado para explicar la patogenia de este síndrome, expondremos aquí únicamente la más aceptada en la actualidad y que ha sido propuesta entre otros por Weiner, Page, Fulton y Reid, después de muchas investigaciones sobre animales. Ellos han logrado reproducir el sindrome de afibrinogenemia en perros, conejos y ratones con la inyección endovenosa de extractos fluídos de placenta o de líquido amniótico y en otras ocasiones traumatizando la placenta en animales embarazados. Con estas investigaciones y otros estudios biológicos sobre el contenido intrauterino han llegado a la conclusión de que tanto la placenta como la decidua y el líquido amniótico son extraordinariamente ricos en tromboplastina. En algunas circunstancias anormales esta tromboplastina sería forzada dentro de la circulación materna e iria a trastornar el equilibrio entre las sustancias procoagulantes y anticoagulantes de la sangre, transformando primero la protrombina en trombina y esta a su turno convirtiendo el fibrinógeno en fibrina. La fibrina se depositaría en forma diseminada en los pequeños vasos formando coágulos o "embolos", llegando a producir en los casos más marcados oclusión de la circulación en los riñones, pulmones, cerebro y otras vísceras vitales. 
Esta utilización de fibrinógeno para la formación de fibrina producirá un marcado descenso o aún ausencia total de aquel en la sangre circulante, dándole a esta el carácter de incoagulable. Se ha hablado también de que esta deplesión de fibrinógeno sea producida por una fibrinolisina o enzima fibrinolítica, pues se ha logrado demostrar la presencia de estas sustancias en algunos casos del síndrome, pero actualmente se cree que ellas son producidas por la lesión del endotelio vascular debida a los depósitos de fibrina o que simplemente se producen en los casos de Stress o de Shock muy marcado, pero ya como una consecuencia y no como causa de la afibrinogenemia.

El mecanismo de salida de la tromboplastina del útero varía en las tres entidades que pueden producir el sindrome: 1) En los casos de desprendimiento prematuro de la placenta probablemente tiene su origen en los tejidos alterados de la placenta separada y en la decidua vecina y pasaría a los senos venosos de sitio en donde se produjo el desprendimiento, debido al aumento de la presión intrauterina por la formación de un coágulo retroplacentario de cierto tamaño; 2) En los casos de embolía o infusión por líquido amniótico, este pasaría a la circulación materna. por uno de estos mecanismos: a) Después de la ruptura de las membranas fetales el líquido podría insinuarse entre estas y la pared uterina para alcanzar el margen de la placenta y entrar a los senos venosos; b) Las venas endocervicales durante el trabajo normal se desgarran ligeramente y aquellas colocadas en la parte más baja del segmento no estarían cubiertas por las membranas fetales cuando estas se han roto prematuramente o al comienzo del trabajo. En los casos de trabajos muy fuertes y rápidos, el exceso de presión intrauterina haría entrar el líquido dentro de estas venas cervicales descubiertas; 3) En el caso de retención prolongada del feto muerto, la tromboplastina se produciría por autolisis de tejido placentario o decidual: en algunos casos la placenta se devitaliza hasta llegar a la liquefacción y éstos serían los más propicios para la producción de material defribinante. Se cree que en este grupo de pacientes la tromboplastina pasaría a la circulación materna debido a las contracciones uterinas, ya sean de trabajo verdadero o del tipo de BraxtonHicks. 


\section{DIAGNOSTICO POR MEDIO DEL LABORATORIO}

Como hemos visto a lo largo de este estudio, el síndrome de afibrinogenemia está caracterizado por una marcada reducción y en ocasiones completa desaparición del fibrinógeno circulante. Existen algunos otros cambios hematológicos como son una pequeña baja en la concentración de protrombina y ocasionalmente la aparición de una fibrinolisina circulante, pero ellos no son de importancia para el diagnóstico del sindrome. Se considera que la concentración normal de fibrinógeno en el plasma es de 250 a 400 miligramos por 100 c. c. y que el nivel crítico es de $100 \mathrm{mgs}$ \%. Por debajo de esta cifra se debe esperar siempre una falla en el mecanismo de coagulación.

Cuando se sospeche la presencia de afibrinogenemia en casos de desprendimiento prematuro de la placenta, embolía de liquido amniótico y retención prolongada de feto muerto o en cualquier caso de hemorragia obstétrica sin explicación aparente, inmediatamente se procede a tomar una muestra de sangre venosa y mientras el laboratorio realiza la prueba cuantitativa da fibrinógeno en el plasma, se hace el más simple de los medios de diagnóstico que el "test de observación del coágulo". Para esto se depositan de 5 a 10 c. c. de sangre en un tubo de los de serología (10 cms. por $12 \mathrm{mms}$ ) y se observa durante 10 o 15 minutos. Si al cabo de este tiempo la sangre no ha coagulado o ha formado un coágulo inestable, se puede concluir que el fibrinógeno circuiante ha disminuído considerabiemente. Si el coágulo se ha formado, se deberá incubar durante una hora a 37 grados centigrados y si este se disuelve, será también debido a hipo o afibrinogenemia.

Otros métodos rápidos y que no necesitan la presencia del laboratorista, son el descrito por Schneider (American Journal of Obstetrics and Ginecology, Vol. 64. No 1) y el descrito por Bonsnes y Sweeney (American Journal of Obstetrics and Gynecology, Vol. 70, Agosto de 1955). Para el primero se hace una serie de diluciones de sangre en solución de Ringer, a las cuales se les puede agregar o no un poco de trombina y se lee el resultado en términos de titulación de fibrina. Los títulos por debajo de 100 sugieren concentraciones de fibrinógeno menores de $100 \mathrm{mgs}$. \%. El segundo método es similar, pero la sangre se debe centrifugar y a! plasma se le agrega solución salina de trombina. Para mayor in- 
formación sobre estos dos métodos se puede consultar al literatura mencionada arriba.

De todas maneras se debe ordenar inmediatamente la concentración de fibrinógeno en el plasma, procedimiento bastante exacto y que el único inconveniente que tiene es que el resultado se demora de una a dos horas. En algunos casos ayudará también el tiempo de protrombina, pues éste se prolonga en los casos de afibrinogenemia.

\section{TRATAMIENTO}

El tratamiento debe ser dirigido a combatir el estado de Shock presentado por la paciente y a restaurar el nivel de fibrinógeno en el plasma. Para lo primero se darán transfusiones, preferentemente de sangre fresca y para lo segundo se administrará fibrinógeno por vía endovenosa. El número de transfusiones que se necesitará en cada caso será determinado por la cantidad de sangre perdida y por el grado de Shock que se presente. La administración de fibrinógeno se iniciará con una dosis de 2 gramos y se seguirá regulando con chequeos periódicos de la sangre cada hora. Para estos chequeos se podrá usar únicamente el "test de observación del coágulo" y mientras se note deficiencia en la coagulación se dará un gramo más de fibrinógeno cada hora. En las publicaciones que se han hecho al respecto se observa que las dosis han oscilado entre 2 y 12 gramos, pero hay innumerables casos que han cedido y 2 y 3 gramos únicamente. Si para los chequeos periódicos de la sangre se ordena la concentración de fibrinógeno en el plasma hay que recordar que el título normal de ésta es de 250 a $400 \mathrm{mgs}$ \%, para regular la administración de fibrinógeno de acuerdo con esta cifra.

El fibrinógeno que se usa para el tratamiento de la afibrinogenemia se ha denominado "fracción 1 de Cohn" y se obtiene de plasma humano; en su preparación se le somete a tratamientos especiales de esterilización para evitar la trasmisión de ictericia por suero homólogo. Se administra por vía endovenosa disuelto en agua destilada o suero glucosado y la velocidad de infusión se regulará de acuerdo con la intensidad del síndrome. No sobra repetir aquí que este es el único tratamiento efectivo de la afibrinogenemia y que las transfusiones de sangre aunque son necesarias para combatir el Shock y restaurar el volumen sanguíneo en ningún caso restablecen los niveles normales de fibrinógeno. Una 
simple transfusión de 500 c. c. de sangre aumentará la concentración del fibrinógeno sanguíneo únicamente en 5 o $10 \mathrm{mgs}$ \%. en una enferma que esté sangrando activamente.

En los casos severos de desprendimiento prematuro de la placenta que se acompañen del síndrome de afibrinogenemia la conducta obstétrica será la misma que se ha venido empleando desde hace muchos años, aceleración del parto, ya sea por inducción o por operación cesárea, pero el pronóstico será mucho más favorable si en el momento de efectuarse la intervención quirúrgica o de provocarse el parto ya se han restaurado los niveles de fibrinógeno sanguíneo.

Una labor profiláctica muy importante se podrá desarrollar en los casos de retención prolongada del feto muerto, especialmente si las pacientes son $\mathrm{Rh}$ negativas y tienen un título alto de anticuerpos. En estos casos se harán chequeos periódicos del fibrinógeno plasmático y se podrá hacer un diagnóstico precoz de afibrinogenemia aún antes de la aparición clínica del síndrome; así el tratamiento se podrá iniciar a tiempo para prevenir la manifestación de la diátesis hemorrágica. Una vez restaurado el nivel de fibrinógeno se procederá cuanto antes a la inducción del parto.

\section{CASOS CLINICOS}

Mientras desempeñaba el cargo de residente en algunos hospitales de los Estados Unidos (Maryland General Hospital y su afiliado Hartford Memorial Hospital, y Lutheran Hospital of Maryland) tuve oportunidad de seguir de cerca 4 casos de afibrinogenemia. Presentaré aquí únicamente dos de ellos por carecer en este momento de la información completa de los otros dos.

Caso número 1.- La enferma de ventiséis años de edad, con antecedente de un embarazo y parto normal a término, tipo O.Rh negativa, fue admitida al Hospital a los $71 / 2$ meses de embarazo a causa de hemorragia nasal profusa y de contracciones uterinas todavia irregulares. Esta enferma había sido vista varias veces en la clínica prenatal y venticinco días antes se le había hecho el diagnóstico de feto muerto; como no presentara complicaciones en aquella época se había resuelto esperar por un parto espontáneo. El título de aglutininas anti-Rh había sido de 1: 340 en el último chequeo. Al examen físico de admisión en el hospital se encontró un pulso de 90 por ciento, tensión arterial 106/70, contracciones uterinas cada 7 minutos, membranas rotas hacía tres horas (se- 
gún información de la enferma), presentación encajada, cuello con un $50 \%$ de borramiento y dilatado un centímetro. La enferma presentaba epístaxis no muy abundante pero continua. Inmediatamente se tomó sangre venosa para el "test de observación del coágulo" y para concentración de fibrinógeno plasmático. A los veinte minutos todavía no se apreciaba formación de cóagulo por lo cual se inició la trasfusión simultánea de dos botellas de sangre mientras se pedía el fibrinógeno al University Hospital en donde estaba centralizado el banco de fibrinógeno de Baltimore. Mientras tanto la enferma continuó en trabajo y a la hora y media de la admisión dio a luz un feto muerto y macerado de $1.300 \mathrm{gms}$. de peso. Inmediatamente después de la expulsión de la placenta, la enferma a pesar de presentar un útero bien contraído y sin desgarros, comenzó a sangrar profusamente. Otros 1.000 c. c. de sangre se iniciaron por vía endovenosa y a los venticinco minutos del parto se empezó la administración de 2 gramos de fibrinógeno disueltos en solución gluconada al 5\%,200 c. c. Una hora después de la administración de este fibrinógeno la enferma todavía presentaba hemorragia nasal y vaginal aunque no tan abundante; al "test de Observación" se vio un coágulo pequeño a los diez minutos, pero este se disolvió por incubación a 37 grados ctgs. Otros dos gramos de fibrinógeno se administraron por vía endovenosa y se iniciaron otros $1.000 \mathrm{c}$. c. de sangre, pues la enferma presentaba ya un buen grado de Shock. A los cinco minutos de iniciada la infusión de fibrinógeno la hemorragia cesó completamente y se apreció la formación de coágulos de buena consistencia.

Los niveles de fibrinógeno plasmático fueron de $30 \mathrm{mgs} . \%$ en la admisión de la paciente, $90 \mathrm{mgs}$. después de la primera dosis de fibrinógeno y $370 \mathrm{mgs}$. \% después de la segunda dosis.

La cantidad de sangre perdida se estimó en 2.000 a 3.000 c. c.. la enferma recibió 3.000 c. c. de sangre (no fresca) y se le administraron cuatro gramos de fibrinógeno. A la enferma se le dio de alta del hospital a los dos días, después de un nuevo chequeo de fibrinógeno ( $420 \mathrm{mgs}$. \%).

Caso número 2.- Una paciente de treinta y un años de edad. Tipo O,Rh positiva con antecedentes obstétricos normales (3 embarazos con partos a término, normales), fue admitida en el hospital, a término, quejándose de dolor abdominal continuo y de hemorragia vaginal más o menos profusa. Al examen físico de admisión el pulso era de 104 por minuto, la tensión arterial 90/60 
y se apreciaba hemorragia vaginal más o menos profusa. Presentaba contracciones uterina fuertes cada cuatro minutos y aunque el útero se apreciaba blando en el intervalo entre las contracciones, la enferma acusaba dolor continuo. No se apreciaron ruidos fetales. Después de un examen vaginal en la sala de cirugía se excluyó la placenta previa y se hizo el diagnóstico de desprendimiento prematuro; en ese examen se encontró una presentación de vértice encajada y el cuello tenía $4 \mathrm{cms}$. de dilatación. Se practicó una amniotomía y la enferma continuó en trabajo y con hemorragia vaginal continua, pero no muy abundante. 500 c. c. de sangre se iniciaron por vía endovenosa. A los treinta minutos, bajo anestesia raquídea en silla, la enferma dio a luz un feto muerto de 3.200 gms. de peso, por medio de forceps de desprendimiento y después de practicar una episiotomía mediana. La placenta fue expulsada inmediatamente después del feto y mostró signos de un desprendimiento prematuro casi completo. Aunque inmediatamente después del parto la enferma se hallaba en buen estado general, el útero estaba bien contraído y no había mayor hemorragia, se le dieron otros 500 c. c. de sangre para reemplazar la que había perdido. A los cuarenta y cinco minutos comenzó a sangrar por la incisión ya suturada de la episiotomía y diez minutos más tarde se manifestó franca hemoragia uterina a pesar del buen tono de la matriz. En ese momento se hizo la prueba de "Observación del coágulo" y se ordenó la prueba de concentración de fibrinógeno. En la primera prueba no se pudo apreciar la formación de ningún coágulo, por lo cual se pidió urgentemente el fibrinógeno y mientras este se obtenía se comenzaron a transfundir 1.000 c. c. de sangre más, pues la tensión arterial ya estaba en 50/20. La enferma siguió sangrando profusamente y sólo cesó la hemorragia algunos minutos después de haber comenzado la infusión de cuatro gramos de fibrinógeno disueltos en 100 c. c. de solución salina. Cuando esta infusión se terminó, ya había pasado completamente la hemorragia.

El nivel de fibrinógeno antes de la administración del tratamiento fue de $23 \mathrm{mgs}$ \% y veinte minutos después de terminada la infusión fue de $290 \mathrm{mgs} \%$.

\section{COMENTARIO}

Como se puede apreciar en la literatura obstétrica de los últimos años, el número de casos de afibrinogenemia aumenta cada día en forma muy notoria; ya hemos visto cómo este sindrome 
constituye una entidad obstétrica definida, con un diagnóstico muy simple y exacto y con un tratamiento bastante agradecido. Hasta donde tengo entendido, en Colombia no se obtiene el fibrinógeno humano o "Fracción 1 de Cohn" que es el único tratamiento efectivo para esta afección. Es por esta razón que quiero aprovechar este trabajo para insinuar que la Sociedad Colombiana de Ginecologia y Obstetricia gestione la introducción de este medicamento al país. Se podría mantener un banco de Fibrinógeno en alguna de las principales clínicas $u$ hospitales de la ciudad, iniciándolo con algunos pocos gramos, que inclusive se podrian obtener gratuitamente por intermedio de alguna de las instituciones médicas norteamericanas. Una vez obtenido el fibrinógeno no sobraría recalcar la necesidad de hacer los chequeos de laboratorio ya descritos, en todos los casos sospechosos de afibrinogenemia y aplicar el medicamento precozmente, con lo cual se salvarian muchas vidas.

\section{BIBLIOGRAFIA}

AMERICAN JOURNAL OF OBSTETRICS AND GYNECOLOGY:

Dieckmann, Volumen 31, página 734, 1936.

Weiner, Reid y Roby, Volumen 60, página 379, 1950.

Weiner, Reid y Roby, Volumen 60, página 1015, 1950.

Crowder, Roy y Bills, Volumen 60, página 1388, 1950.

Marcuse y Duson, Volumen 61, página 695, 1951.

Tunis, Volumen 64, página 72, 1952.

Sluder, Volumen 64, página 118, 1952.

Schneider, Volumen 64, página 141, 1952.

Page y Fulton, Volumen 61, página 1116, 1951.

Schneider, Volumen 63, página 1078, 1952.

Schneider, Volumen 65, página 245, 1953.

Peckham y Middlebrook, Volumen 65, página 644, 1953.

Reid, Weiner y Roby, Volumen 66, página 466, 1953.

Bartholomew, Volumen 66, pág. 1042, 1953.

Stevenson, Braden y Schneider, Volumen 65, 1953.

Schneider, Volumen 69, página 758, 1955.

Heaton y Solomon, Volumen 70, página 320, 1955.

Frick y McKelvy, Volumen 70, página 328, 1955.

Bonsnes y Sweeney, Volumen 70, página 334, 1955.

NEW ENGLAND JOURNAL OF MEDECINE, Moloney y Egan. Vol. 240, página 596, 1949.

JOURNAL OF THE MOUNT SINAI HOSPITAL, Chessin y Greenwald. Volumen 20, página 263, 1953.

ILLINOIS MEDICAL JOURNAL, Skillman y Friedman. Volumen 106, página 329, 1954. 
BRITISH MEDICAL JOURNAL, Barnett y Cussen. Volumen 2, página 676, 1954.

JOURNAL OF THE AMERICAL MEDICAL ASSOCIATION, Volumen 152, página 227, 1953.

SURGICAL CLINICS OF NORTH AMERICA, Weber, Volumen 34, página 1601, 1954.

Dieckmann, TOXEMIAS OF FREGNANCY.

\section{INFORME DE COMISION SOBRE EL TRABAJO DEL Dr. RAFAEL QUIÑONES DAZA}

Bogotá, D. E., noviembre 3 de 1955

Señor Presidente de la Sociedad Colombiana de Obstetricia y Ginecología.- La ciudad.

\section{Señor Presidente:}

Por medio de la presente nos permitimos rendir el informe reglamentario acerca del trabajo titulado "Afibrinogemia en el Embarazo", presentado por el doctor Rafael Quiñones Daza, con el propósito de ingresar como miembro de número a nuestra Sociedad.

El tema escogido por el aspirante no puede ser más novedoso y nos parece que por este solo hecho merecería un concepto favorable, pero además se trata de una monografía que analiza con claridad y sencillez todos' los aspectos conocidos hasta el presente sobre el Síndrome Afibrinogemia e Hipo Fibrinogenemia. El número reducido de observaciones en que se basa el trabajo no 10 demerita, pues sabemos que esta es una entidad que se ha identificado hasta hace relativamente poco tiempo pero que día en día aumentan las publicaciones de nuevos casos; bástanos decir a este respecto que desde la comunicación original de steiner en 1941 sobre Embolía Ammiótica y Afibrinogenemia, sólo se han comprobado 57 casos según una reciente revisión hecha por K. H. Grahan.

Entre nosotros sólo sabemos de un caso en el cual se hizo el diagnóstico positivo mediante el laboratorio; se trataba de una 
enferma sensibilizada con Anticuerpos Anti-Rh y portadora de un feto muerto.

La importancia que tienen los estudios relacionados con estados hemorrágicos en el parto, el embarazo y el puerperio, se debe principalmente a que la hemorragia uterina ocupa el primer lugar como causa de mortalidad materna y dentro de estas hemorragias las producidas por alteraciones de la coagulación habian sido las más desconocidas hasta el presente y especialmente en nuestro medio; de ahí la palpitante actualidad del trabajo que nos ocupa.

El doctor Quiñones hizo el estudio de todo lo concerniente al Síndrome:

$\mathrm{Su}$ historia, estudio clínico, patogenia, diagnóstico de laboratorio y tratamiento, por lo tanto nos parece que para una mejor ilustración le cedamos la palabra al autor.

La insinuación que hace el doctor Quiñones a la sociedad con el fin de gestionar la importación de Fibrinógenos parece de una gran utilidad y creemos que la sociedad debe acogerla y llevarla a la práctica en corto tiempo.

Señor Presidente: nos permitimos solicitar de la sociedad la aprobación del trabajo cuyo estudio se nos ha encomendado y no queremos terminar sin felicitar muy sinceramente a su autor por la manera sencilla y clara como lo ha presentado.

Atentamente, Vuestra Comisión: 